prevalence in antenatal population in Karnataka districts, we analysed data from three such surveys to detect heterogeneity. The three districts analysed here are Belgaum (Northern Karnataka), Bellary (middle of the state) and Mysore (Southern Karnataka).

Methods We conducted a comparative analysis of these three GPS conducted between 2005 and 2007. Subjects were selected using a two-stage cluster sampling design with equal number of rural and urban participants, and of men and women, with a target sample size of 6000 per district. Questionnaires on socio-demographic factors and HIV risk behaviour were administered. Blood samples were tested for HIV, syphilis and HSV-2 antibodies, whereas urine samples were tested for gonorrhoea and chlamydia using nucleic acid amplification tests. A descriptive analysis of prevalence of HIV and sexually transmitted infections (STIs) was conducted according to age, district and place of residence (rural/urban).

Results Abstract P1-S1.13 table 1 shows HIV prevalence per district according to gender and place of residence. Belgaum had the highest overall HIV and HSV-2 prevalence (16.9\%) and the lowest prevalence of curable STIs (Chlamydia- $0.38 \%$; Syphilis- $0.42 \%$ ) among the three districts. Women in Belgaum had a higher HIV prevalence $(\mathrm{OR}=2.16,95 \% \mathrm{CI}-1.02$ to 4.58$)$ compared to women in Mysore. The HIV epidemic in Belgaum is predominantly rural and among women. In Bellary, it is predominantly urban and among men. Mysore had the lowest prevalence of HIV and HSV-2 (10.9\%) and the highest prevalence of curable STIs (Chlamydia-1.05\%; Syphilis-1.38\%) among the three districts. There were only six cases of gonorhoea in total (five in Mysore and one in Bellary).

Abstract P1-S1.13 Table 1 Gender specific prevalence of HIV by district and place of residence

\begin{tabular}{llll}
\hline Place of residence & Belgaum \% $(95 \% \mathbf{C I})$ & Bellary \% $(\mathbf{9 5 \%} \mathrm{Cl})$ & Mysore \% $(95 \% \mathrm{CI})$ \\
\hline All total & $1.43(0.86$ to 2.01$)$ & $1.18(0.74$ to 1.62$)$ & $0.80(0.50$ to 1.09$)$ \\
Males & $1.28(0.51$ to 2.06$)$ & $1.24(0.70$ to 1.78$)$ & $0.98(0.45$ to 1.52$)$ \\
Females & $1.58(0.94$ to 2.23$)$ & $1.13(0.59$ to 1.66$)$ & $0.65(0.28$ to 1.02$)$ \\
& & & \\
Urban total & $0.63(0.18$ to 1.09$)$ & $1.36(0.65$ to 2.07$)$ & $0.94(0.49$ to 1.39$)$ \\
Urban males & $0.69(0.25$ to 1.12$)$ & $1.64(0.71$ to 2.56$)$ & $1.06(0.35$ to 1.78$)$ \\
Urban females & $0.58(0.00$ to 1.16$)$ & $1.12(0.37$ to 1.86$)$ & $0.84(0.34$ to 1.35$)$ \\
& & & \\
Rural total & $1.69(0.90$ to 2.49$)$ & $1.05(0.46$ to 1.64$)$ & $0.71(0.31$ to 1.10$)$ \\
Rural males & $1.48(0.41$ to 2.55$)$ & $0.97(0.29$ to 1.65$)$ & $0.93(0.13$ to 1.74$)$ \\
Rural females & $1.90(1.01$ to 2.79$)$ & $1.13(0.34$ to 1.93$)$ & $0.51(0.00$ to 1.05$)$ \\
\hline
\end{tabular}

${ }^{*}$ All figures in weighted percentages, $95 \% \mathrm{Cl}$.

Conclusion The HIV epidemic in Karnataka shows considerable heterogeneity. This analysis validates the observed north-south gradient. The sex work structure in these three districts might explain the heterogeneity of the HIV epidemic in these three districts. Higher prevalence of HIV and HSV-2 and lower prevalence of curable STIs in Belgaum suggests a late epidemic phase. Mysore may have an early phase epidemic with higher prevalence of curable STIs.

\section{P1-S1.14 IS HIV PREVALENCE DECLINING IN SOUTHERN INDIA? EVIDENCE FROM TWO ROUNDS OF GENERAL POPULATION SURVEYS IN BAGALKOT DISTRICT, KARNATAKA}

\section{doi:10.1136/sextrans-2011-050108.14}

\begin{abstract}
${ }^{1} \mathrm{~S}$ P Rajaram, ${ }^{2} \mathrm{~J}$ E Bradley, 'B M Ramesh, ' $\mathrm{S}$ Isac, ${ }^{1} \mathrm{R}$ G Washington, ${ }^{3} \mathrm{~S}$ Moses, ${ }^{3} \mathrm{~J}$ F Blanchard, ${ }^{3} \mathrm{M}$ Becker, ${ }^{2} \mathrm{M}$ Alary. ${ }^{1}$ Karnataka Health Promotion Trust, Bangalore, India; ${ }^{2}$ Centre hospitalier affilié universitaire de Québec, Quebec, Canada; ${ }^{3}$ University of Manitoba, Winnipeg, Canada
\end{abstract}

Background As a part of the evaluation of Avahan, the India AIDS initiative of the Bill \& Melinda Gates Foundation, a cross-sectional survey was undertaken in the general population of Bagalkot district, Karnataka state, South India, in 2009. This replicated an earlier survey in 2003 that had examined HIV prevalence and risk behaviours.

Methods The repeat survey in 2009 was conducted in the same rural and urban areas as the 2003 study. The study covered 10 rural villages and 20 urban blocks of three of the six talukas (sub-district units) in the district. In both surveys, a target sample of 6600 adult males and females was selected. Urine and blood samples were collected from all consenting participants for HIV and STI testing. An individual was deemed HIV positive, if positive on two different tests. We compared HIV and STI prevalence in 2003 and 2009. We also examined the age-specific distribution of HIV prevalence among rural and urban males and females at both time points. These analyses used logistic regression that considers survey design to adjust for characteristics of the population that may have changed between the two rounds.

Results Overall, HIV prevalence in the district declined from $3.16 \%$ in 2003 to $2.58 \%$ in 2009, although this decline was not statistically significant $(\mathrm{OR}=0.82, \mathrm{p}=0.278)$. The prevalence of active syphilis was $<1 \%$ and was similar in both periods. A slight decline in the prevalence of HSV-2 was observed over the period, but the difference was not statistically significant. There was a significant decline observed in HIV prevalence among persons aged 15-24, among persons below 30 years in urban areas, and among all women younger than 20 years of age (Abstract P1-S1.14 table 1). Among rural males aged 40 and above, we observed a significant increase in HIV prevalence from 0.71 to 5.33 (Abstract P1-S1.14 table 1), a level similar to that found in the younger age group in 2003.

Abstract P1-S1.14 Table 1 HIV prevalence and adjusted OR among selected population groups

\begin{tabular}{lllll}
\hline Sub-group & $\begin{array}{l}\text { Round 1, } \\
\text { 2003 (\%) }\end{array}$ & $\begin{array}{l}\text { Round 2, } \\
\mathbf{2 0 0 9}(\%)\end{array}$ & $\begin{array}{l}\text { Adjusted 0R, 2009 vs } \\
\mathbf{2 0 0 3 ~ ( 9 5 \% ~ C l ) ~}\end{array}$ & p Value \\
\hline Overall & 3.16 & 2.58 & $0.82(0.57$ to 1.18$)$ & 0.278 \\
Age 15-24 & 2.4 & 1.26 & $0.54(0.30$ to 0.96$)$ & 0.038 \\
Age 15-29, urban & 2.04 & 0.77 & $0.36(0.20$ to 0.63$)$ & 0.001 \\
Age 15-19, female & 2.25 & 0.49 & $0.15(0.04$ to 0.61$)$ & 0.009 \\
Age 40+, rural males & 0.71 & 5.33 & $13.80(2.59$ to 73.45$)$ & 0.006 \\
\hline
\end{tabular}

Conclusion We observed a downward overall trend in HIV prevalence in Bagalkot district over the period examined, and a significant decline in HIV prevalence among younger age groups, where HIV prevalence more closely reflects incidence. The increase in HIV prevalence among older rural males may have been due to a cohort effect, as they aged over time. This may also have in part reflected increased survival in those age groups, as a result of the scaling-up of antiretroviral treatment programs throughout the state.

\section{P1-S1.15 THE STATUS OF THE HIV EPIDEMIC IN LEBANON-SYSTEMATIC REVIEW AND SYNTHESIS}

doi:10.1136/sextrans-2011-050108.15

${ }^{1} \mathrm{G}$ Mumtaz, ${ }^{2} \mathrm{~N}$ Hilmi, ${ }^{1} \mathrm{~L}$ Abu-Raddad. ${ }^{1}$ Weill Cornell Medical College in Qatar, Doha, Oatar; ${ }^{2}$ World Bank, USA

Background The Middle East and North Africa (MENA) continues to be perceived as a region with limited HIV epidemiological data. The objective of this work was to review and synthesise for the first time all available data related to HIV in Lebanon, in order to assess the status of the epidemic in this country and provide the basis for future interventions, prevention, and research needs. 